\title{
Neurophysiological Mechanisms Involved in Transfer of Procedural Knowledge
}

\author{
Monica A. Perez, ${ }^{1}$ Steven P. Wise, ${ }^{2}$ Daniel T. Willingham, ${ }^{3}$ and Leonardo G. Cohen ${ }^{1}$ \\ ${ }^{1}$ Human Cortical Physiology Section, National Institute of Neurological Disorders and Stroke and ${ }^{2}$ Laboratory of Systems Neuroscience, National Institute \\ of Mental Health, National Institutes of Health, Bethesda, Maryland 20892, and ${ }^{3}$ Department of Psychology, University of Virginia, Charlottesville, Virginia \\ 22904
}

Learning to perform a motor task with one hand results in performance improvements in the other hand, a process called intermanual transfer. To gain information on its neural mechanisms, we studied this phenomenon using the serial reaction-time task (SRTT). Sixteen, right-handed volunteers trained a 12-item sequence of key presses repeated without the subjects' knowledge. Blocks with no repeating sequence, called random blocks, were interspersed with sequence-training blocks. Response times improved in random and training blocks in both hands. The former result reflects nonspecific improvement in performance, and the latter represents a sequence-specific improvement. To evaluate changes in the primary motor cortex (M1), we tested resting motor thresholds (RMT), recruitments curves to transcranial magnetic stimulation (RC), short intracortical inhibition (SICI), and interhemispheric inhibition (IHI) from the dominant left (learning) to the nondominant right (transfer) hemisphere, before and after SRTT training. Training resulted in (1) increased RC and decreased SICI but no changes in RMT in the learning hemisphere, (2) decreased SICI and no changes in RC or RMT in the transfer hemisphere, and (3) decreased IHI. The amount in IHI after training correlated with nonspecific performance improvements in the transfer hand but not with sequence-specific performance improvements. Our results indicate that modulation of interhemispheric inhibition between the M1 areas may, as a result of the learning that has occurred in one hemisphere after practice with one hand, contribute to faster, more skilled performance of the opposite hand.

Key words: motor cortex; intermanual transfer; interhemispheric inhibition; transcallosal pathways; motor learning; intracortical inhibition

\section{Introduction}

Procedural learning has been extensively studied using the serial reaction-time task (SRTT). In this test, subjects are instructed to respond to visual cues by pressing different keys on a keyboard. The order of key presses repeats a predetermined sequence, typically without the subjects' knowledge. The result is that the response time (RT) in the learning hand shortens even when the subject remains unaware of the existence of a repeating sequence. Previous studies identified the involvement of an extensive network that includes the supplementary motor area, primary sensorimotor cortex, parietal regions, cerebellum, and basal ganglia in the performance of this task (Hazeltine et al., 1997; Grafton et al., 1998; Honda et al., 1998; Willingham et al., 2002; Daselaar et al., 2003; Bischoff-Grethe et al., 2004). The primary motor cortex (M1) of the learning hemisphere, i.e., the hemisphere contralateral to the hand performing the task, is particularly active during

\footnotetext{
Received Sept. 20, 2006; revised Dec. 2, 2006; accepted Dec. 20, 2006.

This work was supported by the intramural program of the National Institute of Neurological Disorders and Stroke.

We thank Drs. Robert Chen, Orlando Swayne, and Satoshi Tanaka for their helpful comments on this manuscript. Correspondence should be addressed to Leonardo G. Cohen, Human Cortical Physiology Section, National Institute of Neurological Disorders and Stroke, National Institutes of Health, 10 Center Drive, Bethesda, MD 20892-1430. E-mail: cohen!@ninds.nih.gov.

D0I:10.1523/JNEUROSCI.4128-06.2007

Copyright $\odot 2007$ Society for Neuroscience $\quad$ 0270-6474/07/271045-09\$15.00/0
}

SRTT, as measured by transcranial magnetic stimulation (TMS) (Pascual-Leone et al., 1994), electroencephalography (Zhuang et al., 1998), and positron emission tomography (Hazeltine et al., 1997; Grafton et al., 1998; Honda et al., 1998). So is, to some extent, the M1 ipsilateral to the learning hand (Daselaar et al., 2003; Bischoff-Grethe et al., 2004).

Studies in both humans and nonhuman animals have demonstrated that the procedural knowledge acquired in one hand transfers to the other hand (Parlow and Kinsbourne, 1989; Howard and Howard, 1997; Rand et al., 1998, 2000; Grafton et al., 2002; Japikse et al., 2003). For SRTT, the neural substrates underlying this form of intermanual transfer (Grafton et al., 2002; Japikse et al., 2003) may involve M1 (Grafton et al., 2002). Because activation of the corpus callosum has been reported and linked to intermanual transfer of sensory and motor information (Tettamanti et al., 2002; Omura et al., 2004), it is not surprising that split-brain and acallosal patients show less transfer (Lassonde et al., 1995; de Guise et al., 1999). The way in which the two M1 areas interact in the process of intermanual transfer of procedural knowledge is not known. Neurophysiological changes in M1 of the transfer hemisphere or in the transcallosal interaction between M1 areas could support this transfer process.

The purpose of this investigation was to characterize functional changes in $\mathrm{M} 1$ of both hemispheres associated with intermanual transfer of procedural motor learning, as assessed by the 
SRTT. To that end, we tested various measures of motor cortical excitability. Resting motor thresholds (RMT) and recruitments curves (RC) to TMS reflect excitability of high- and lowthreshold motor cortical neurons to the stimulating magnetic coil (Siebner and Rothwell, 2003). Short intracortical inhibition (SICI) is a measure that is thought to reflect activity in intracortical GABAergic inhibitory interneurons (Kujirai et al., 1993; Ziemann et al., 1996). Interhemispheric inhibition (IHI) from the left (learning) to the right (transfer) M1 likely reflects activity predominantly in interhemispheric glutamatergic connections that synapse over local GABAergic interneurons in the opposite hemisphere (Ferbert et al., 1992; Gerloff et al., 1998a; Di Lazzaro et al., 1999).

\section{Materials and Methods}

Subjects. Sixteen healthy volunteers (nine females, seven males) with an average age of $28 \pm 7$ years participated in the study. All subjects gave their informed consent to the experimental procedure, which was approved by the local ethics committee. The study was performed in accordance with the Declaration of Helsinki. All subjects were right handed and trained with the right hand for $\sim 30 \mathrm{~min}$ on the SRTT. Two of the 16 subjects had experience playing a musical instrument. None of them was, at the time of the study, regularly practicing any activity that involved sequential finger movements.

SRTT and transfer test. Subjects were seated in an armchair with both arms flexed at the elbow by $90^{\circ}$ and the wrist in a neutral position. They practiced a modified version of the SRTT (Nissen and Bullemer, 1987) presented on a computer screen using a custom script based on SuperLab (version 2.0; Cedrus, San Pedro, CA). The GO signal was displayed on a computer screen (one asterisk and three dots evenly spaced horizontally, all appearing simultaneously). The position of the asterisk, which varied among the four possible locations each time a display appeared, indicated the required key press. Subjects were instructed to respond as fast and accurately as possible to the presentation of the asterisk by pressing one of the four keys with the appropriate finger (index finger for key 1 , middle finger for key 2, ring finger for key 3 , and little finger for key 4). After the correct key was pressed, the next display of three dots and one asterisk appeared in the computer screen. If subjects made an error by pressing an incorrect key, the program enforced a delay until the correct key was pressed. We measured RTs defined as the time interval between the GO signal and the correct key button press.

Subjects performed a total of 20 blocks of key presses, separated by resting periods in each experimental session (Fig. $1 A$ ). Each subject participated in only one session. Each block was composed of 10 repetitions of a 12 -item sequence for a total of 120 key presses per block. Subjects began by performing the task with their right hands, and they were initially presented with two random blocks (R1 and R2) (see the part of Fig. $1 \mathrm{~A}$ for right-hand performance). In these and all subsequent random blocks, the asterisk appeared in a randomly selected series of locations instead of in a predetermined repeating sequence. The first random block (R1) was used for familiarizing each subject with the task, and the second (R2) was used to measure the initial performance improvements achieved by that experience. In blocks 3-7 and 9-13 (Fig. 1A), called practice or training blocks, subjects were presented with a predetermined repeating sequence (sequence $A$, which was 4-2-3-1-1-3-2-1-3-4-2-4, as expressed in terms of asterisk and key locations). We label each block according to both its order and the sequence used. For example, A1 is the third block presented overall (after R1 and R2), and it is the first block with sequence A. Blocks 8 and 14 were also random (R3 and R4, right hand) (Fig. 1A). After block 14, participants were asked "Did you notice anything special about the task?" and "Do you have anything to report regarding the task?". Subjects who reported that they noticed a sequence or a repeating pattern were asked to describe it in as much detail as possible, and the information was written for later analysis.

After the 14 blocks of performance with their right hands, subjects were tested on performance with their left hand. They were presented with two random blocks (R1 and R2 for the left hand) (Fig. 1 A). As with

\section{A}

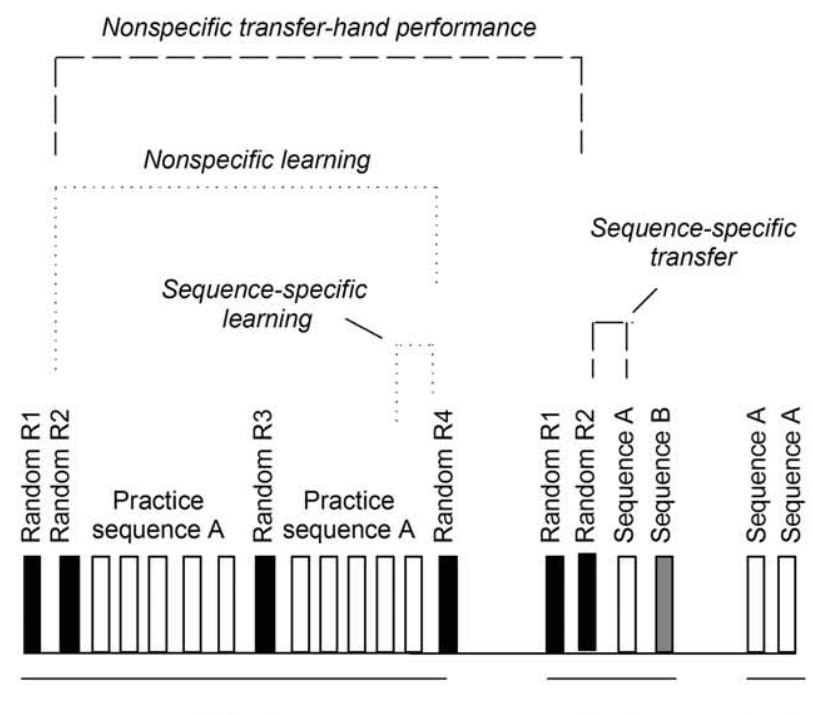

\section{Right hand Left hand Right hand}

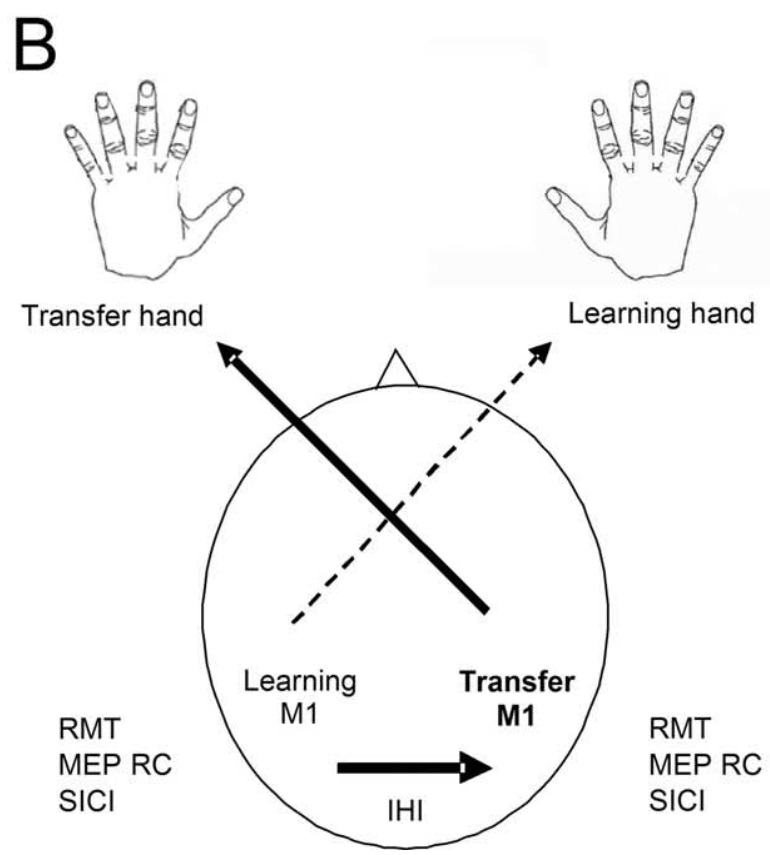

Figure 1. Experimental design. $A$, Diagram showing the order of the sequence and random (R) blocks practiced with the right and left hand. For the right hand, procedural learning that was sequence specific was calculated as the difference in RT between the last sequence block (A10) and the last random block (R4) (Willingham et al., 2000), and learning that was nonspecific to the sequence was calculated as the difference in RT between the right-hand random blocks R2 and R4 (dotted lines). For the left hand, sequence-specific transfer was calculated as the difference in RT between the sequence (A) and random block (R2) and nonspecific performance as the difference in RT between the R2 random blocks in the right and left hands (dashed lines). $B$, Diagram showing measures of motor cortical excitability tested before and after the SRTT.

the right-hand training, $\mathrm{R} 1$ was used to familiarize the subjects with performing the task with their left hands, and R2 was used to measure performance. The two random blocks were followed by two sequence blocks, involving sequences A and B (Fig. $1 A$ ). Sequence A (4-2-3-1-1- 
A

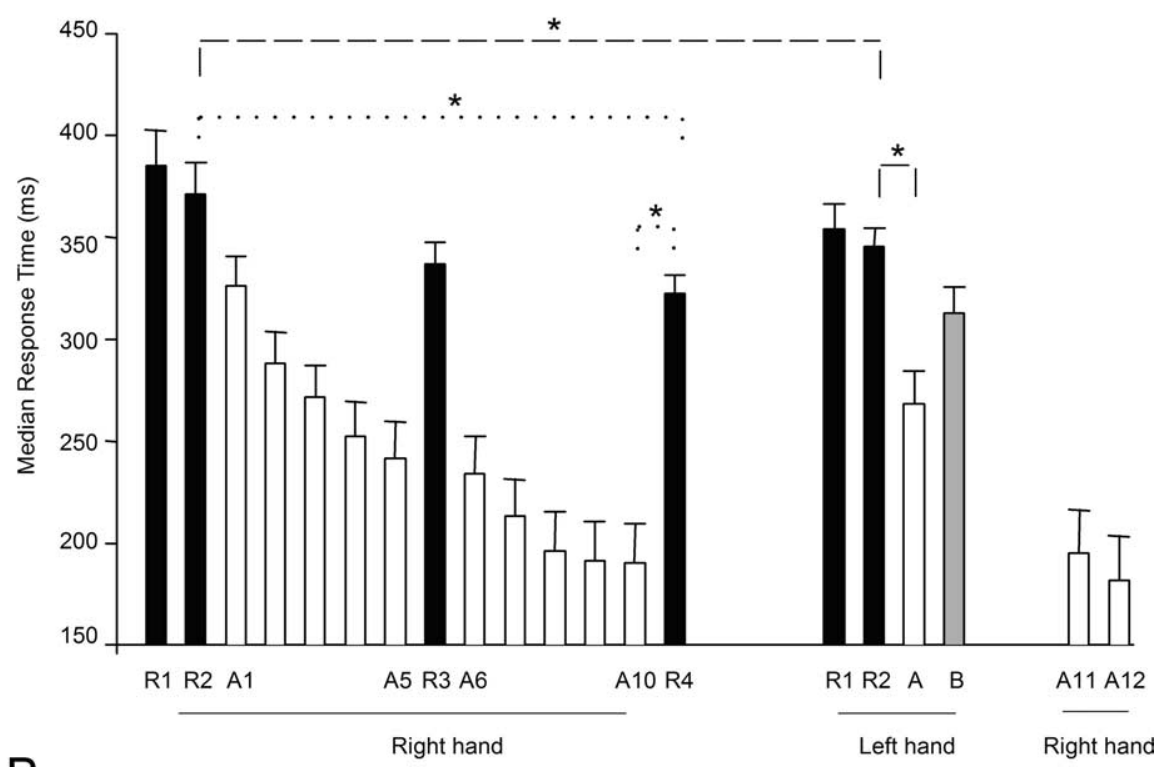

B

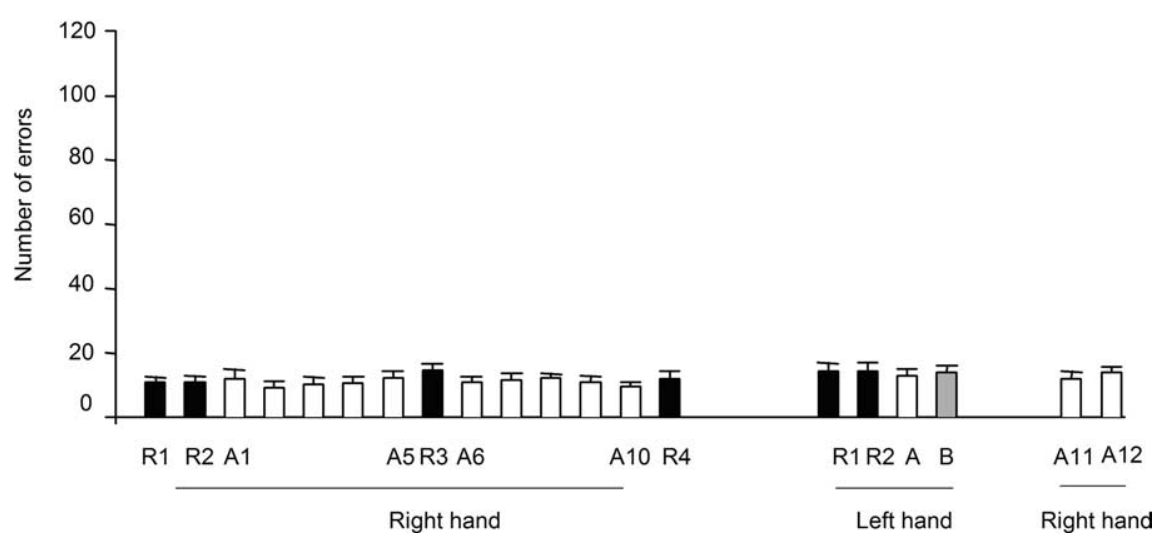

Figure 2. Response time $(\boldsymbol{A})$ and mean errors $(\boldsymbol{B})$ in random and sequence blocks during the SRTT. $\boldsymbol{A}$, The abscissa shows block type and performing hand in temporal order, and the ordinate shows response times. Note the progressive shortening of response times in random ( $R 1-R 4$, right hand) and sequence $A$ ( $A 1-A 10$, right hand) blocks during performance of the SRTT with the right hand, maintained at the end of the experiment (A11, $A 12$, right hand). Performance of the same sequence $A$ with the left hand showed shorter response times compared with both unpracticed sequence B (gray bar) and random blocks. Sequence-specific transfer and nonspecific transfer-hand performances are shown in dotted lines for the right-hand and left transfer-hand performance in dashed lines. $\boldsymbol{B}$, Mean number of accuracy errors (wrong key presses) with the right and left hand. The abscissa shows the order of all random and sequence blocks practiced with the right and left hand. Note the stable overall accuracy during the course of the experiment. Variance is expressed as $\mathrm{SE}_{;}{ }^{*} p<0.05$.

3-2-1-3-4-2-4) was the same sequence as that presented to the right hand in terms of asterisk and key-press position, with the proviso that the position of the asterisk was for each display the mirror image of that one used for the right hand. Thus, the asterisk in position 4 instructed the subject to press the fourth key on the keyboard with the left little finger. Sequence B (1-3-3-4-2-1-3-2-4-1-4-2) was a new unlearned sequence. After completing these four blocks with the left hand, subject proceeded to perform two more sequence A blocks with the right hand (Fig. $1 \mathrm{~A}$, last two blocks) to determine stability of performance gains.

For the right hand, procedural sequence-specific learning was calculated as the difference in RT between the last sequence block (A10) and the last random block (R4) (Willingham et al., 2000), and nonspecific was calculated as the difference in RT between the right-hand random blocks R2 and R4 (Fig. $1 \mathrm{~A}$, dotted lines). For the left hand, sequencespecific transfer was calculated as the difference in RT between the sequence block (A) and random block (R2), and nonspecific transfer-hand performance was calculated as the difference in RT between the random blocks $\mathrm{R} 2$ for the right and left hands (Fig. $1 \mathrm{~A}$, dashed lines).

Electromyographic recordings. Surface electrodes were positioned bilaterally on the skin overlying the first dorsal interosseous (FDI) muscles in a bipolar montage (interelectrode distance, $2 \mathrm{~cm}$ ). The amplified EMG signals were filtered (bandpass, $25 \mathrm{~Hz}$ to $1 \mathrm{kHz}$ ), sampled at $2 \mathrm{kHz}$, and stored on a personal computer for off-line analysis.

Transcranial magnetic stimulation. TMS was delivered to the optimal scalp position for activation of the FDI muscles overlying left- and right-hand primary motor cortex. Motorevoked responses (MEPs) were elicited by TMS stimuli delivered from a Magstim 200 stimulator (Magstim company, Dyfed, UK) through a figure-of-eight coil (loop diameter, $8 \mathrm{~cm}$; type number, SP15560). Measures of cortical excitability, including the resting motor threshold (RMT), defined as the lowest intensity of TMS output required to evoke MEPs of at least 50 $\mu \mathrm{V}$ in peak-to-peak amplitude in at least three of five consecutive trials (Rossini et al., 1994), MEP RCs, SICIs as tested with the paired-pulse technique, and IHI from the left to the right hemisphere, were tested preceding and immediately after the experimental protocol described above (Fig. 1B). Because of the length of the physiological determinations and to avoid excessive fatigue, RMT, RC, and SICI were tested in 6 of the 16 subjects participating in the behavioral experiments and RMT and IHI in the other 10. All electrophysiological measurements were taken before and after the 30 min of training with the right hand.

$M E P$ recruitment curves. Stimulus intensities started at 5\% below RMT and increased in 5\% steps of maximal device output until the MEP amplitude did not experience additional increases or the maximal stimulator output was reached. Each RC determination was repeated twice. Therefore, there were 10 trials available to determine MEP amplitudes at each stimulus intensity. MEP amplitudes were measured peak-to-peak, averaged off-line, and expressed as a percentage of the maximal motor response (M-max). To determine M-max, the ulnar nerve was stimulated ( $1 \mathrm{~ms}$ rectangular pulse) with supramaximal intensity using bipolar surface electrodes placed at the wrist. Subsequently, data were normalized to the individual RMT of each participant. To this end, mean baseline activity for each recruitment curve was calculated, and values 1 $\mathrm{SD}$ above the baseline were included in a regression line. RMT values were determined by the interaction between the $x$ intercept and the mean baseline using the following linear regression formula: $y=a+b x$. All measurements were expressed as a percentage of the FDI M-max. Therefore, $\mathrm{RC}$ values are expressed relative to M-max responses and RMT for each individual.

SICI. SICI was tested bilaterally using the method described by Kujirai et al. (1993). A conditioning stimulus (CS) was set at 70\% of the RMT. This low-intensity stimulus does not activate corticospinal fibers and does not produce changes in the excitability of spinal motoneurons (Di Lazzaro et al., 2001). The test stimulus (TS) was adjusted to produce an MEP of $\sim 1 \mathrm{mV}$ at rest. TS was delivered $2.5 \mathrm{~ms}$ after CS, an optimal interstimulus interval for eliciting SICI and to avoid mixture of the two phases of inhibition (Fisher et al., 2002). Fifteen TS and CS were pre- 
sented randomly at each time interval, and responses were recorded for off-line analysis. Stimuli were applied every $5 \mathrm{~s}$. Measurements were repeated three times before the training session until a consistent baseline was established. After the training was completed, two determinations were done 1 min apart.

IHI. IHI was tested after a randomized conditioning-test design reported previously (Ferbert et al., 1992). A suprathreshold CS set at $120 \%$ of the RMT was given $10 \mathrm{~ms}$ before a TS delivered to the contralateral side. The CS was always given to the left (learning) motor cortex and the TS to the right (transfer) motor cortex (Fig. $1 B$ ). The coil was positioned at the optimal location for activating the left and right FDI, respectively. The two coils were secured by straps and attached to a coil holder to ensure that the same area of the primary motor cortex was stimulated. The handle of the coils pointed backwards and laterally $\sim 45^{\circ}$ to the midsagittal line. Previous evidence has shown that the strength of IHI is modified by changes in the size of the TS and by the intensity of the CS (Ferbert et al., 1992; Daskalakis et al., 2002). Therefore, the intensity for the TS was adjusted to produce an MEP of three different sizes at rest: test MEP1 $(0.34 \pm 0.12 \mathrm{mV})$, test MEP2 (0.86 \pm $0.21 \mathrm{mV})$, and test MEP3 $(1.41 \pm 0.82 \mathrm{mV})$. The order of the testing was randomized. In addition, in five of the subjects, we measured the effect of changing stimulus intensity of the CS $(1.0,1.1,1.15,1.2$, and 1.3 RMT) on the strength of IHI. Fifteen TS and CS were averaged during off-line analysis. Stimuli were applied every $5 \mathrm{~s}$. Measurements were repeated three times at each CS intensity tested before the training session until a consistent baseline was established. After the transfer test was completed, two measurements were taken at each of the conditions tested 1 min apart.

Data analysis. Repeated-measures ANOVA was used to evaluate the median RT and the number of errors in the right hand with sequence blocks (A1-A10) and random blocks (R2-R4) as factor. A paired $t$ test was used to evaluate procedural sequencespecific learning with the right hand by comparing the median RT between A10 and R4 (Willingham et al., 2000). To assess transfer of sequence-specific learning, a paired $t$ test was used to compare the median RT in the R2 and sequence A blocks practiced with the left hand. To assess nonspecific transfer, a paired $t$ test was used to compare the median RT in the R2 blocks practiced with the left and right hand. A $t$ test was used to compare performance improvements in those subjects who notice and did not notice some sequence of targets after training.

Repeated-measures ANOVA test was used to determine the effect of time (before and after) and stimulus intensity (1.0, 1.1, 1.2, 1.3, 1.4, 1.5, 1.6, and 1.7) on MEP amplitudes and the effect of time (before and after) and hemisphere (left and right) on RMT. We tested the effect of time (before and after), test MEP amplitude (test MEP1, test MEP2, and test MEP3) and conditioned MEP intensity (1.0, 1.1, 1.15, 1.2, and 1.3 RMT) on IHI. We evaluated the effect of time (before and after training) and hemisphere (left and right) on SICI. A paired $t$ test was used to compare M-max and size of TS before and after training. Significance was set at $p<0.05$. Variance is expressed as mean \pm SEM. Pearson's correlation analysis was used to test correlations as needed. A
Table 1. RMT in left and right FDI muscle measured before (Pre) and after (Post) SRTT training in both hemispheres expressed as a percentage of the stimulator output

Learning hemisphere (right FDI) Transfer hemisphere (left FDI)

Pre Post Pre Post

\begin{tabular}{|c|c|c|c|c|}
\hline RMT & $40 \pm 1.9 \%$ & $38.6 \pm 3 \%$ & $42.9 \pm 1.6 \%$ & $41.5 \pm 1.1 \%$ \\
\hline Test MEP1 & & & $0.34 \pm 0.05 \mathrm{mV}$ & $0.37 \pm 0.04 \mathrm{mV}$ \\
\hline Test MEP2 & & & $0.86 \pm 0.06 \mathrm{mV}$ & $0.72 \pm 0.07 \mathrm{mV}$ \\
\hline Test MEP3 & & & $1.41 \pm 0.04 \mathrm{mV}$ & $1.39 \pm 0.14 \mathrm{mV}$ \\
\hline IHI MEP1 & & & $41.1 \pm 6.6 \%$ & $67 \pm 7.8 \%$ \\
\hline IHI MEP2 & & & $58.2 \pm 6.4 \%$ & $82.6 \pm 7.3 \%$ \\
\hline IHI MEP3 & & & $57.0 \pm 5.8 \%$ & $65.6 \pm 2.6 \%$ \\
\hline
\end{tabular}

The amplitude of three different test MEP size (test MEP1, test MEP2, and test MEP3) elicited in the right hemisphere during evaluation of IHI. Notice that the
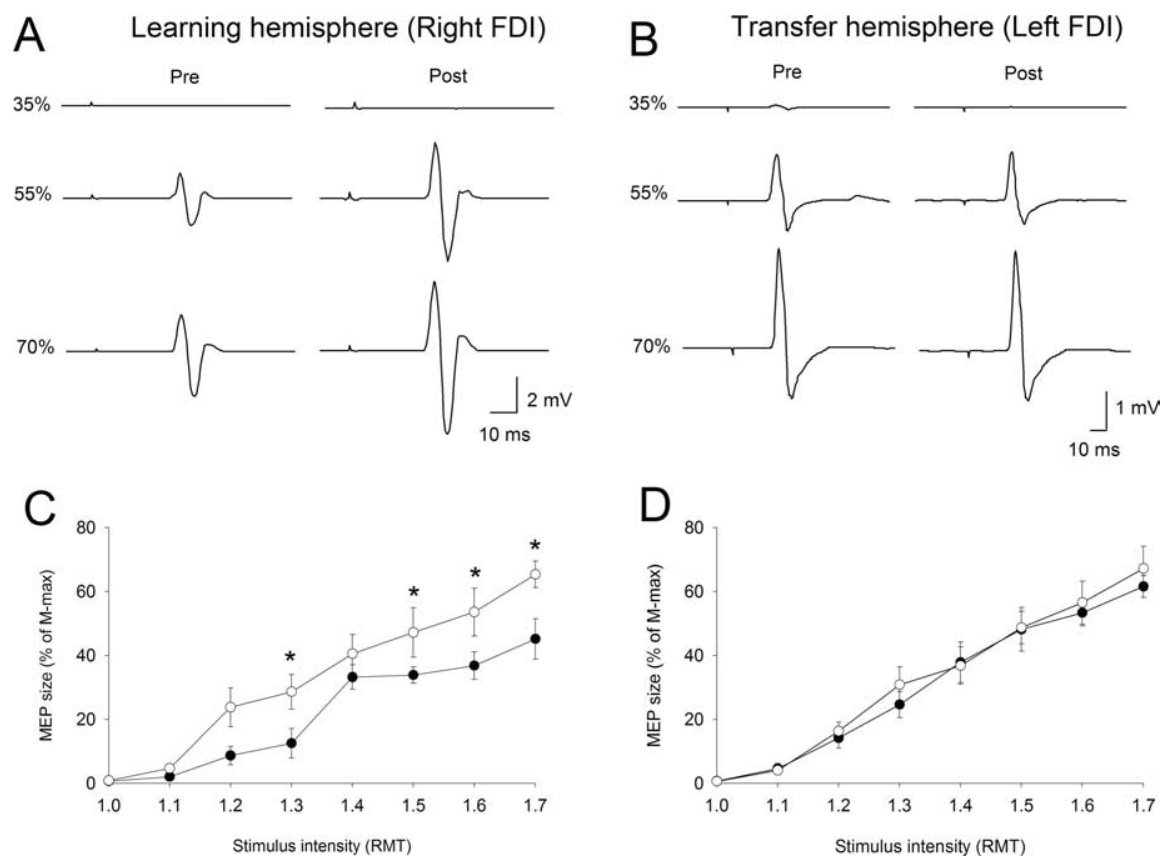

Figure 3. MEP recruitment curves. $A, B, M E P$ amplitudes from FDI muscles $(A, B)$ before and after (pre and post) training on the SRRT task with stimulation of the left $(\boldsymbol{A})$ and right $(\boldsymbol{B})$ motor cortices in a single subject. Note the increase in MEP amplitudes in the earning hemisphere after the SRTT. C and $\boldsymbol{D}$ show the recruitment curves group data indicating the effect of SRTT training with the (ive before (filled circles) and after (open circles) the SRTT training. Note the increase in recruitment curves in the learning hemisphere in the absence of changes in the transfer hemisphere. Error bars indicate SEs; ${ }^{*} p<0.05$.

\section{Results}

All subjects completed the SRTT training with the right hand $(n=16)$. Nine of the 16 subjects did not notice any particular pattern during practice of the task. Seven of the 16 subjects reported that some sequence of targets repeated during the SRTT training, but none was able to reproduce the sequence.

\section{Right (learning) hand}

Repeated-measures ANOVA showed a significant effect of random block on RT ( $F=16.8 ; p \leq 0.001)$. Post hoc analysis showed shorter RT in the last random block R4 (322 $\pm 9.4 \mathrm{~ms})$ relative to the first random block R2 ( $371 \pm 15.3 \mathrm{~ms} ; p \leq 0.001)$ (Fig. $2 A$ ), indicating a nonspecific performance improvement that was unrelated to the repeating sequence. In addition to the changes in random blocks, ANOVA showed a significant effect of sequence block on RT ( $F=43.4 ; p \leq 0.001)$. Post hoc analysis showed shorter RT in the last sequence block A10 (190 $\pm 19.4 \mathrm{~ms})$ relative to the first sequence block A1 (326 $\pm 14.7 \mathrm{~ms} ; p=0.004)$ (Fig. 
2A). A significant difference was found between the RT in blocks $\mathrm{A} 10$ and R4 (A10, $190 \pm 19.4 \mathrm{~ms} ; \mathrm{R} 4,322 \pm 9.4 \mathrm{~ms} ; p \leq 0.001)$, indicating a significant procedural sequence-specific learning with the right hand (Fig. 2 A). No differences in sequence-specific learning with the right hand were found between subjects who noticed or did not notice some sequence of targets after training $(p=0.7)$.

Repeated-measures ANOVA showed no effect of sequence block $(F=0.4 ; p=0.8)$ or random block $(F=1.4 ; p=0.2)$ on the number of errors performed by the right hand (Fig. $2 B$ ).

\section{Left (transfer) hand}

There was a significant difference between RT in blocks R2 and A in the left hand (R2, $345 \pm 9.4 \mathrm{~ms} ; \mathrm{A}, 268 \pm 16.3 ; p \leq 0.001$ ) (Fig. $2 A$ ), indicating a significant transfer of sequence-specific procedural learning to the left hand. One consequence of this transfer was that the subjects reached in only one block the level of performance that had previously taken three blocks when learning the sequence with the right hand, despite the fact that the subjects performed the transfer test with their nondominant hand. Performance improvements in the left hand were more prominent with sequence $A(R 2-A, 77 \pm 12.2 \mathrm{~ms})$ than with the unpracticed sequence B (R2-B, $33 \pm 9.8 \mathrm{~ms} ; p<0.001)$. Further substantiating this transfer effect, performance improvements from R2 to A in the left hand (R2-A, $77 \pm 12.2 \mathrm{~ms}$ ) were larger than performance improvements from $\mathrm{R} 2$ to $\mathrm{A} 1$ in the right hand (R2-A1, $45 \pm 7.1 \mathrm{~ms} ; p \leq 0.01$ ), a finding present in 11 of the 16 subjects tested. Subjects who noticed or did not notice some sequence of targets achieved similar sequence-specific learning with their left hand $(n=0.4)$.

Additionally, there was a significant difference between RT in block R2 practiced with the left (R2 left, $345 \pm 9.2 \mathrm{~ms})$ and right (R2 right, $371 \pm 15.3 \mathrm{~ms} ; p=0.005$ ) (Fig. $2 A$ ) hands, pointing to a significant nonspecific improvement of the skill to the left hand, present in 14 of 16 subjects. No differences were found between the subjects who noticed or did not notice some sequence of targets $(p=0.3)$.

\section{MEP recruitment curves}

Repeated-measures ANOVA showed no effect of time $(F=0.81$; $p=0.4)$ or hemisphere $(F=0.86 ; p=0.3)$ on FDI RMTs (Table $1)$. ANOVA showed a significant effect of time $(F=9.22 ; p=$ $0.02)$, intensity $(F=62.7 ; p \leq 0.001)$, and their interaction time and intensity $(F=5.5 ; p \leq 0.001)$ on MEP amplitudes in the right FDI muscle. Post hoc testing showed a significant increase in right FDI MEP amplitude at $1.3(p=0.01), 1.5(p=0.03), 1.6(p=$ 0.02 ), and 1.7 ( $p \leq 0.001$ ) of the RMT (Fig. $3 A, C$ ). For the left FDI, there was a significant effect of intensity $(F=55.6 ; p \leq$ 0.001 ) but not time or their interaction (Fig. $3 B, D$ ). M-max amplitude did not change over time in either left $(p=0.5)$ or right $(p=0.3)$ FDI.

\section{SICI}

Repeated-measures ANOVA showed a significant effect of time $(F=33.08 ; p=0.002)$ but not hemisphere or their interaction on SICI. The results of no difference in SICI between left-right hemispheres are consistent with previous studies (Cicinelli et al., 2000; Maeda et al., 2002). Post hoc analysis revealed a significant attenuation of SICI [ (conditioned MEP $\times 100) /$ test MEP] on the right (mean pretraining, $44.5 \pm 6.3 \%$; mean posttraining, $67.7 \pm$ $6.2 \% ; p=0.01$ ) (Fig. $4 A$ ) and left (mean pretraining, $46.5 \pm$ $7.6 \%$; mean posttraining, $85.6 \pm 18 \%$; $p=0.02$ ) (Fig. $4 B$ ) FDIs. Test MEP amplitudes were corrected at the end of training to

\section{A Learning hemisphere (Right FDI)}
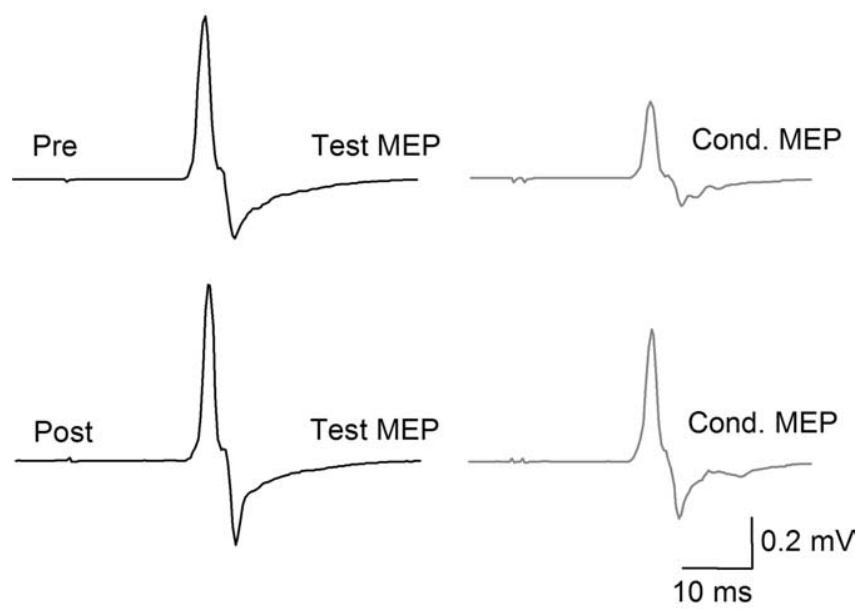

\section{B Transfer hemisphere (Left FDI)}
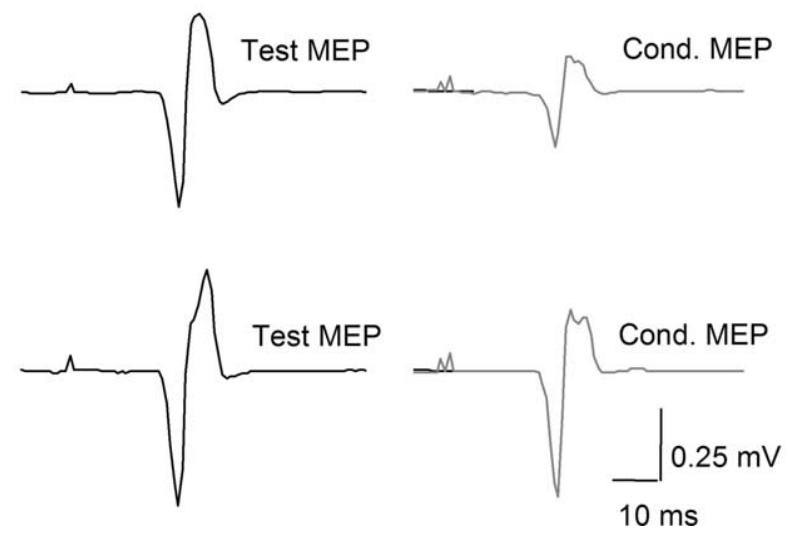

Figure 4. Short intracortical inhibition. SICI $(2.5 \mathrm{~ms})$ in $\mathrm{FDI}(\boldsymbol{A}, \boldsymbol{B})$ measured as the ratio between [(conditioned MEP $\times 100)$ /test MEP] before (Pre) and after (Post) performance of the SRTT task with stimulation of the learning $(\boldsymbol{A})$ and transfer $(\boldsymbol{B})$ hemisphere in a single subject. Note the presence of disinhibition in both learning and transfer hemispheres.

match values pretraining (left FDI: mean pretraining, $1.21 \pm$ $0.14 \mathrm{mV}$; mean posttraining, $1.03 \pm 0.11 \mathrm{mV}$; NS; right FDI: mean pretraining, $0.90 \pm 0.1 \mathrm{mV}$; mean posttraining, $0.84 \pm$ $0.09 \mathrm{mV}$; NS).

\section{IHI}

IHI was evaluated at three different test MEP amplitudes (Table 1). At baseline, there was deeper IHI with test MEP1 $(0.34 \pm 0.05$ $\mathrm{mV}$ ) than with the other two test MEP sizes evaluated ( $p=0.01$ and $p=0.02$, respectively) (Fig. $5 A$, Table 1 ). These results are in agreement with previous studies showing that, at a larger size of a test MEP, the magnitude of IHI is decreased (Ferbert et al., 1992; Daskalakis et al., 2002). We observed no differences in the magnitude of IHI between test MEP2 and test MEP3, and this is also in agreement with previous results (Gilio et al., 2003).

Repeated-measures ANOVA showed a significant effect on IHI of time $(F=17.3 ; p=0.002)$ and test MEP amplitude $(F=$ $3.8 ; p=0.04)$ but not their interaction $(F=1.6 ; p=0.2)$ (Fig. 
$5 A, B)$. The CS intensities were adjusted to elicit MEPs of $\sim 1.0 \mathrm{mV}$ (pretraining, $0.90 \pm 0.2$; posttraining, $0.96 \pm 0.2$; NS), accomplished with $51.9 \pm 7.4 \%$ (pre) and $50.4 \pm 6.3 \%$ (post) of the output of the stimulator, respectively (NS). There was no significant effect of time on test MEP amplitudes $(F=0.69 ; p=0.42)$ (Table 1$)$. In a control experiment, aimed at determining the influence of changing CS stimulus intensities $(n=5)$ on IHI, repeatedmeasures ANOVA showed a significant effect of time $(F=7.9 ; p=0.04)$ and CS intensity $(F=18.4 ; p \leq 0.001)$ on IHI in the absence of interaction. Posttraining attenuation of IHI was identified at various CS intensities (Fig. 5C). Repeatedmeasures ANOVA showed no effect of TIME $(F=0.29 ; p=0.6)$, MEP size $(F=$ $1.2 ; p=0.3)$, or their interaction $(F=1.4$; $p=0.28)$ on test MEP amplitudes in this control experiment.

\section{Correlation analysis}

Overall, subjects who performed the random and sequence blocks faster with the right (learning) hand by the end of the training were also the fastest with the left hand on the transfer test $(r=0.75, p \leq$ 0.001 ; and $r=0.77, p \leq 0.001$ respectively). Subjects who were faster performing random blocks with the left (transfer) hand at the end of the training were the ones with less IHI after training $(r=0.66$; $p=0.03)$ (Fig. 6A). This correlation was not present with IHI values obtained before training $(r=0.4 ; p=$ $0.2)$. No correlation was found between sequence-specific transfer (R2-A) and IHI (Fig. 6B), SICI, M-max, or MEP threshold, or between nonspecific transfer hand performance and SICI, M-max, or MEP threshold. In addition, no correlation was found between nonspecific (R2 left hand) and sequencespecific (R2-A) performance improvements in the transfer hand $(r=-0.1 ; p=0.6)$.

\section{Discussion}

We studied the effects of SRTT training on functional changes in $\mathrm{M} 1$ in an intermanual transfer task. Behaviorally, both sequencespecific and nonspecific performance improvements occurred, and the main electrophysiological findings were that learning resulted in (1) larger RC and decreased SICI in the learning hemisphere, (2) unchanged RC and decreased SICI in the transfer hemisphere, and (3) decreased IHI from the learning to the transfer hemisphere. After training, the amount of IHI correlated with nonspecific performance improvements in the transfer hand.

\section{Nonspecific and sequence-specific performance improvements}

The present results confirm that RT improves with SRTT training in both random and sequence blocks and in both the learning and transfer hands (Willingham, 1999; Willingham et al., 2000, 2002; Grafton et al., 2002; Japikse et al., 2003). Nonspecific improvements in performance, as seen in random blocks, probably reflect learning to optimize the procedure for selecting the correct key

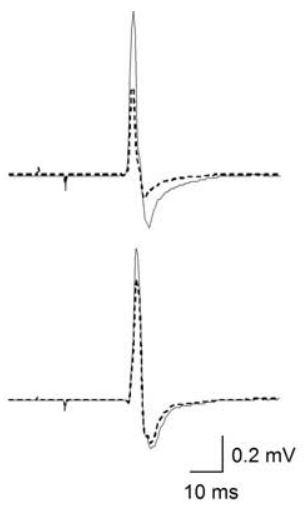

C

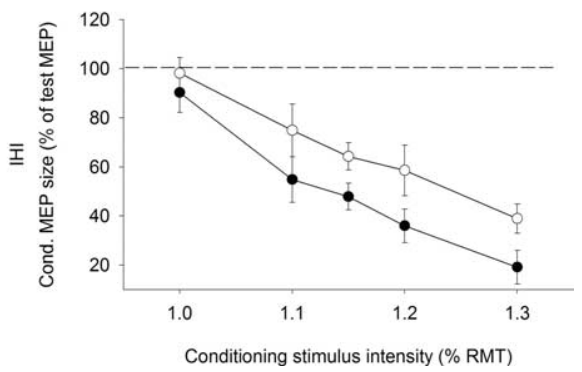

Conditioning stimulus intensity (\% RMT)

Figure 5. Interhemispheric inhibition. $\boldsymbol{A}, \mathrm{IHI}$ at $10 \mathrm{~ms}$ from a single subject recorded before (Pre) and after (Post) the SRTT task. Test MEPs are shown in solid lines and conditioned MEPs in dotted lines. Note the well defined IHI before training (Pre) at the three test MEP amplitudes (test MEP1, test MEP2, and test MEP3; see Materials and Methods) and the relative disinhibition shown after training (Post). $\boldsymbol{B}$, Group data $(n=10)$. The abscissa shows the three test MEP amplitudes evaluated $(0.34 \pm 0.05,0.86 \pm 0.06$, practice of the SRTT, identifiable at all three test MEP amplitudes. $\boldsymbol{C}$, Influence of changing conditioning stimulus intensities (expressed as percentage of RMT) on the magnitude of IHI. Note the existence of attenuation in IHI (from filled circles to open circles) at all intensities above RMT. Error bars indicate SEs; ${ }^{*} p<0.05$.

after stimulus presentation, as well as developing the general skill necessary to implement the key press (Willingham, 1999). The magnitude of nonspecific changes was small compared with the sequence-specific improvements, indicating that subjects could not predict the upcoming cues in random blocks. The larger, sequence-specific improvements reflected learning both nonspecific aspects and the order of key-press locations (Willingham, 1999).

\section{Neurophysiological findings}

Learning hemisphere

Corticospinal excitability, as assessed by the amplitude of the MEPs, increased in the absence of changes in RMTs. This finding agrees with previous studies after finger-sequence training (Pascual-Leone et al., 1994, 1995). In the learning hemisphere, we also observed a decrease in SICI similar to that reported by Classen et al. (1999) and by Liepert et al. (1998) after training on tasks engaging fine control of hand muscles. In humans, several studies showed that modulation of SICI accompanies practice-dependent plasticity in the hand area of M1 (Liepert et al., 1998; Classen et al., 1999; Nordstrom and Butler, 2002).

\section{Transfer hemisphere}

No previous experiments have studied the influence of the SRTT on neurophysiological function of the hemisphere ipsilateral to a learning hand, referred to here as transfer hemisphere. We found that MEP amplitudes and RMTs did not change, but SICI decreased. Practice of simple sequential thumb opposition 


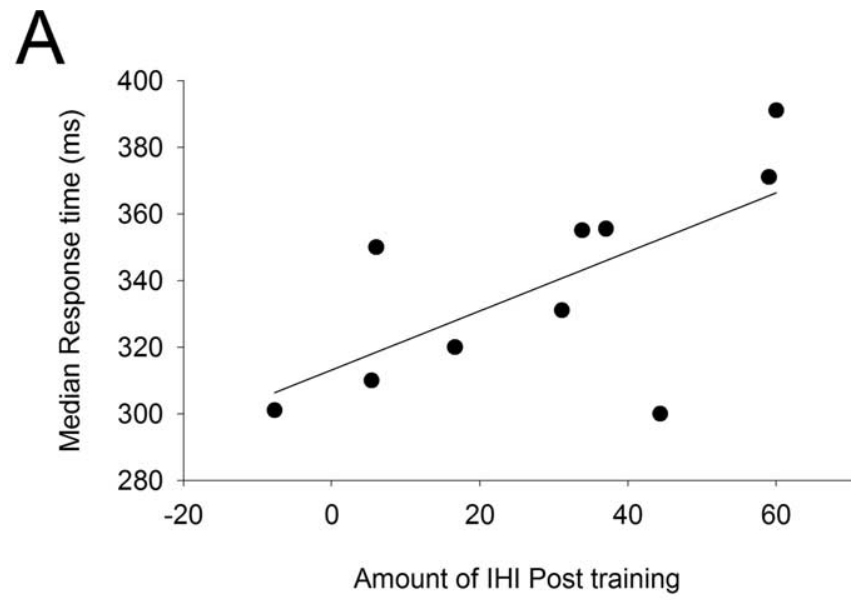

B

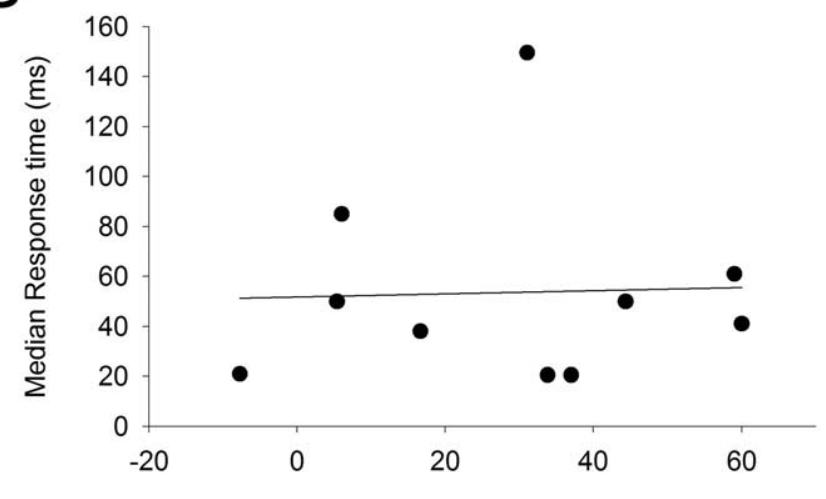

Amount of $\mathrm{IHI}$ Post training

Figure 6. Correlations. $\boldsymbol{A}$, The abscissa indicates the magnitude of IHI in which the size of the conditioned MEP is expressed as a percentage of the size of test MEP amplitude. The ordinate shows the median RT in random block (R2) practiced with the left (transfer) hand (nonspecific transfer-hand performance). Note that the decrease in RT is associated with a decrease in IHI. $\boldsymbol{B}$, The abscissa is as in $\boldsymbol{A}$. The ordinate shows the median RT of the difference between blocks R2 and $A$ practiced with the left hand (sequence-specific transfer). Note that there was no relationship between RT and IHI.

movements results in increase MEP amplitudes in the hemisphere ipsilateral to the trained hand (Tinazzi and Zanette, 1998). Pioneering studies (Rossini et al., 1985, 1987) and more recent investigations (Muellbacher et al., 2000; Hortobagyi et al., 2003) have shown that voluntary movements in one hand lead to nonspecific facilitatory effects on cortical excitability of the ipsilateral hemisphere. Thus, it appears that motor activity in one hand leads to excitability increases in the hemisphere ipsilateral to the moving hand. Neuroimaging studies showed bilateral M1 activation after SRTT training (Daselaar et al., 2003; Bischoff-Grethe et al., 2004), which might reflect excitatory or inhibitory activity (Waldvogel et al., 2000; Almeida and Stetter, 2002). The decrease in SICI in our study could result from a form of interhemispheric facilitation from the learning hemisphere, as proposed by HundGeordiadis and von Cramon (1999), and neurophysiological evidence for interhemispheric facilitatory pathways exists (Hanajima et al., 2001). Alternatively, these neuroimaging changes could reflect the presence of interhemispheric inhibitory interactions or a combination of both (Nudo and Masterton, 1986; Almeida and Stetter, 2002). There was no correlation between changes in SICI in the transfer hemisphere and transfer hand performance.

\section{IHI from the learning to the transfer hemisphere}

Attenuation of a test MEP by a conditioning pulse applied to the opposite hemisphere reflects predominantly transcallosal interactions (Ferbert et al., 1992; Di Lazzaro et al., 1999), with some possible spinal contribution (Gerloff et al., 1998a). Possible pathways mediating these transcallosal effects include glutamatergic projections from one M1 to the other, acting through local GABAergic interneurons (Berlucchi, 1990; Meyer et al., 1995; Chen, 2004). We found a decrease in IHI, which by the end of training correlated with the nonspecific transfer hand performance. This finding suggests that IHI between the M1 areas may play a role in general aspects of the transfer hand performance: a less inhibited cortex could generate outputs faster, thus leading to lower RTs. However, our findings do not support a crucial role of IHI in sequence-specific transfer, which more likely depends on interactions among hierarchically higher motor regions (Hikosaka et al., 1999; Bapi et al., 2000; Daselaar et al., 2003; BischoffGrethe et al., 2004). The lack of correlation between nonspecific and sequence-specific performance of the transfer hand supports the idea that these two aspects are mediated by different mechanisms.

Is it possible that decreased SICI or increased MEP in the learning hemisphere conditioned the described changes in IHI (Chen, 2004)? TMS stimulus intensities applied as conditioning shocks to the learning hemisphere were adjusted to elicit comparable MEPs before and after training, indicating that the net physiological effect of the TMS pulse on corticomotoneuronal connections was comparable. In terms of the influence of SICI on IHI, the presence of SICI in one hemisphere results in less IHI directed toward the opposite hemisphere (R. Chen, personal communication).

MEP amplitudes elicited by the test shock applied to the transfer hemisphere did not change (Table 1) and therefore could not explain changes in IHI. We found that SICI was decreased in the transfer hemisphere. Could this change in SICI impact our findings of decreased IHI? Daskalakis et al. (2002) showed that a conditioning TMS pulse applied to one hemisphere results in decreased SICI tested $10 \mathrm{~ms}$ later in the opposite hemisphere. Chen (2004) suggested that different local intracortical inhibitory interneurons mediate SICI and IHI. More work is required to characterize in detail the interactions between SICI and IHI.

IHI decreases with SRTT training might result from downregulation of M1 transcallosal inhibitory activity, mediated through changes in temporal or spatial summation in transcallosal pathways. This, in turn, would affect their efficacy on local inhibitory circuits. As a result, the effective action of transcallosal fibers recruited by a given conditioning stimulus could change.

\section{Functional considerations}

Our findings suggest that transcallosal interactions between M1 areas may contribute to nonspecific aspects of performance in the transfer hand, particularly to optimizing the timing of visuomotor processing. This proposal agrees with findings pointing to the importance of transcallosal influences on motor execution (Thut et al., 1997; Murase et al., 2004; Duque et al., 2005a), including assisting bilateral movements (Andres et al., 1999) and suppressing unwanted motor activity in the opposite limb during unilateral movements (Duque et al., 2005b). Thus, the involvement of IHI between M1 areas may vary according to behavioral requirements.

Although our results do not support a direct role of M1 in sequence-specific transfer, we cannot exclude this possibility entirely because M1 may contribute to some forms of sequential 
movements (Gerloff et al., 1998b; Hikosaka et al., 1999; Bapi et al., 2000; Ashe et al., 2006). Furthermore, neurons in the motor cortex seem to encode general movements and sequential aspects of actions concurrently (Ben-Shaul et al., 2004). Accordingly, different components of a motor sequence might be channeled into separate but interacting systems, which may reflect differences in the strength and distribution of callosal connections (Rouiller et al., 1994) or the functional role of specific areas (Ashe et al., 2006).

As for mechanism, recent evidence suggests that practice influences interhemispheric inhibitory interactions between the M1 areas, which change with the execution of a learned task (Shim et al., 2005). Furthermore, long-term practice, such as professional musicians undertake, is associated with a reduction of IHI (Ridding et al., 2000). Together with our data (Fig. 6A), these findings suggest that this decreased IHI could support general aspects of motor performance rather than the specific skill being learned. The nonspecific features involved in this process are those in which the M1 is heavily engaged, including generation and timing of fast finger movements (Davare et al., 2006; Verstynen et al., 2006). Perhaps the reduction of IHI facilitates processing in the transfer hemisphere (Huda et al., 1999). If so, then, like other local intracortical inhibitory processes (Floeter and Rothwell, 1999; Reynolds and Ashby, 1999), the circuitry mediating IHI may also play a role in modulating the cortical outputs that produce intended movements. The generalized performance improvement induced by this modulation could form a foundation for the sequence-specific procedural knowledge contributed by higher-level motor areas.

\section{References}

Almeida R, Stetter M (2002) Modeling the link between functional imaging and neuronal activity: synaptic metabolic demand and spike rates. NeuroImage 17:1065-1079.

Andres FG, Mima T, Schulman AE, Dichgans J, Hallett M, Gerloff C (1999) Functional coupling of human cortical sensorimotor areas during bimanual skill acquisition. Brain 122:855-870.

Ashe J, Lungu OV, Basford AT, Lu X (2006) Cortical control of motor sequences. Curr Opin Neurobiol 16:213-221.

Bapi RS, Doya K, Harner AM (2000) Evidence for effector independent and dependent representations and their differential time course of acquisition during motor sequence learning. Exp Brain Res 132:149-162.

Ben-Shaul Y, Drori R, Asher I, Stark E, Nadasdy Z, Abeles M (2004) Neuronal activity in motor cortical areas reflects the sequential context of movement. J Neurophysiol 91:1748-1762.

Berlucchi G (1990) Commisurotomy studies in animals. In: Handbook of neurophysiology, Vol 4 (Boller F, Grafman J, eds), pp 9-47. Amsterdam: Elsevier.

Bischoff-Grethe A, Goedert KM, Willingham DT, Grafton ST (2004) Neural substrates of response-based sequence learning using fMRI. J Cogn Neurosci 16:127-138.

Chen R (2004) Interactions between inhibitory and excitatory circuits in the human motor cortex. Exp Brain Res 154:1-10.

Cicinelli P, Traversa R, Oliveri M, Palmieri MG, Filippi MM, Pasqualetti P, Rossini PM (2000) Intracortical excitatory and inhibitory phenomena to paired transcranial magnetic stimulation in healthy human subjects: differences between the right and left hemisphere. Neurosci Lett 288:171-174.

Classen J, Liepert J, Hallett M, Cohen LG (1999) Plasticity of movement representation in the human motor cortex. Electroencephalogr Clin Neurophysiol Suppl 51:162-173.

Daselaar SM, Rombouts SA, Veltman DJ, Raaijmakers JG, Jonker C (2003) Similar network activated by young and old adults during the acquisition of a motor sequence. Neurobiol Aging 24:1013-1019.

Daskalakis ZJ, Christensen BK, Fitzgerald PB, Roshan L, Chen R (2002) The mechanisms of interhemispheric inhibition in the human motor cortex. J Physiol (Lond) 543:317-326.

Davare M, Duque J, Vandermeeren Y, Thonnard JL, Olivier E (2006) Role of the ipsilateral primary motor cortex in controlling the timing of hand muscle recruitment. Cereb Cortex 17:353-362.

de Guise E, del Pesce M, Foschi N, Quattrini A, Papo I, Lassonde M (1999) Callosal and cortical contribution to procedural learning. Brain 122:1049-1062.

Di Lazzaro V, Oliviero A, Profice P, Insola A, Mazzone P, Tonali P, Rothwell JC (1999) Direct demonstration of interhemispheric inhibition of the human motor cortex produced by transcranial magnetic stimulation. Exp Brain Res 124:520-524.

Di Lazzaro V, Oliviero A, Profice P, Meglio M, Cioni B, Tonali P, Rothwell JC (2001) Descending spinal cord volleys evoked by transcranial magnetic and electrical stimulation of the motor cortex leg area in conscious humans. J Physiol (Lond) 537:1047-1058.

Duque J, Hummel F, Celnik P, Murase N, Mazzocchio R, Cohen LG (2005a) Transcallosal inhibition in chronic subcortical stroke. NeuroImage 28:940-946.

Duque J, Mazzocchio R, Dambrosia J, Murase N, Olivier E, Cohen LG (2005b) Kinematically specific interhemispheric inhibition operating in the process of generation of a voluntary movement. Cereb Cortex 15:588-593.

Ferbert A, Priori A, Rothwell JC, Day BL, Colebatch JG, Marsden CD (1992) Interhemispheric inhibition of the human motor cortex. J Physiol (Lond) 453:525-546.

Fisher RJ, Nakamura Y, Bestmann S, Rothwell JC, Bostock H (2002) Two phases of intracortical inhibition revealed by transcranial magnetic threshold tracking. Exp Brain Res 143:240-248.

Floeter MK, Rothwell JC (1999) Releasing the brakes before pressing the gas pedal. Neurology 53:664-665.

Gerloff C, Cohen LG, Floeter MK, Chen R, Corwell B, Hallett M (1998a) Inhibitory influence of the ipsilateral motor cortex on responses to stimulation of the human cortex and pyramidal tract. J Physiol (Lond) 510:249-259.

Gerloff C, Corwell B, Chen R, Hallett M, Cohen LG (1998b) The role of the human motor cortex in the control of complex and simple finger movement sequences. Brain 121:1695-1709.

Gilio F, Rizzo V, Siebner HR, Rothwell JC (2003) Effects on the right motor hand-area excitability produced by low-frequency rTMS over human contralateral homologous cortex. J Physiol (Lond) 551:563-573.

Grafton ST, Hazeltine E, Ivry RB (1998) Abstract and effector-specific representations of motor sequences identified with PET. J Neurosci 18:9420-9428.

Grafton ST, Hazeltine E, Ivry RB (2002) Motor sequence learning with the nondominant left hand. A PET functional imaging study. Exp Brain Res $146: 369-378$

Hanajima R, Ugawa Y, Machii K, Mochizuki H, Terao Y, Enomoto H, Furubayashi T, Shiio Y, Uesugi H, Kanazawa I (2001) Interhemispheric facilitation of the hand motor area in humans. J Physiol (Lond) 531:849-859.

Hazeltine E, Grafton ST, Ivry R (1997) Attention and stimulus characteristics determine the locus of motor-sequence encoding. A PET study. Brain 120:123-140

Hikosaka O, Nakahara H, Rand MK, Sakai K, Lu X, Nakamura K, Miyachi S, Doya K (1999) Parallel neural networks for learning sequential procedures. Trends Neurosci 22:464-471.

Honda M, Deiber MP, Ibanez V, Pascual-Leone A, Zhuang P, Hallett M (1998) Dynamic cortical involvement in implicit and explicit motor sequence learning. A PET study. Brain 121:2159-2173.

Hortobagyi T, Taylor JL, Petersen NT, Russell G, Gandevia SC (2003) Changes in segmental and motor cortical output with contralateral muscle contractions and altered sensory inputs in humans. J Neurophysiol 90:2451-2459.

Howard Jr JH, Howard DV (1997) Age differences in implicit learning of higher order dependencies in serial patterns. Psychol Aging 12:634-656.

Huda K, Salunga TL, Chowdhury SA, Kawashima T, Matsunami K (1999) Dopaminergic modulation of transcallosal activity of cat motor cortical neurons. Neurosci Res 33:33-40.

Hund-Georgiadis M, von Cramon DY (1999) Motor-learning-related changes in piano players and non-musicians revealed by functional magnetic-resonance signals. Exp Brain Res 125:417-425.

Japikse KC, Negash S, Howard Jr JH, Howard DV (2003) Intermanual transfer of procedural learning after extended practice of probabilistic sequences. Exp Brain Res 148:38-49. 
Kujirai T, Caramia MD, Rothwell JC, Day BL, Thompson PD, Ferbert A, Wroe S, Asselman P, Marsden CD (1993) Corticocortical inhibition in human motor cortex. J Physiol (Lond) 471:501-519.

Lassonde M, Sauerwein HC, Lepore F (1995) Extent and limits of callosal plasticity: presence of disconnection symptoms in callosal agenesis. Neuropsychologia 33:989-1007.

Liepert J, Classen J, Cohen LG, Hallett M (1998) Task-dependent changes of intracortical inhibition. Exp Brain Res 118:421-426.

Maeda F, Gangitano M, Thall M, Pascual-Leone A (2002) Inter- and intraindividual variability of paired-pulse curves with transcranial magnetic stimulation (TMS). Clin Neurophysiol 113:376-382.

Meyer BU, Roricht S, Grafin von Einsiedel H, Kruggel F, Weindl A (1995) Inhibitory and excitatory interhemispheric transfers between motor cortical areas in normal humans and patients with abnormalities of the corpus callosum. Brain 118:429-440.

Muellbacher W, Facchini S, Boroojerdi B, Hallett M (2000) Changes in motor cortex excitability during ipsilateral hand muscle activation in humans. Clin Neurophysiol 111:344-349.

Murase N, Duque J, Mazzocchio R, Cohen LG (2004) Influence of interhemispheric interactions on motor function in chronic stroke. Ann Neurol 55:400-409.

Nissen MJ, Bullemer P (1987) Attentional requirements of learning: evidence from performance measures. Cognit Psychol 19:1-32.

Nordstrom MA, Butler SL (2002) Reduced intracortical inhibition and facilitation of corticospinal neurons in musicians. Exp Brain Res 144:336-342.

Nudo RJ, Masterton RB (1986) Stimulation-induced $\left[{ }^{14} \mathrm{C}\right] 2$-deoxyglucose labeling of synaptic activity in the central auditory system. J Comp Neurol 245:553-565.

Omura K, Tsukamoto T, Kotani Y, Ohgami Y, Minami M, Inoue Y (2004) Different mechanisms involved in interhemispheric transfer of visuomotor information. NeuroReport 15:2707-2711.

Parlow SE, Kinsbourne M (1989) Asymmetrical transfer of training between hands: implications for interhemispheric communication in normal brain. Brain Cogn 11:98-113.

Pascual-Leone A, Grafman J, Hallett M (1994) Modulation of cortical motor output maps during development of implicit and explicit knowledge. Science 263:1287-1289.

Pascual-Leone A, Nguyet D, Cohen LG, Brasil-Neto JP, Cammarota A, Hallett M (1995) Modulation of muscle responses evoked by transcranial magnetic stimulation during the acquisition of new fine motor skills. J Neurophysiol 74:1037-1045.

Rand MK, Hikosaka O, Miyachi S, Lu X, Miyashita K (1998) Characteristics of a long-term procedural skill in the monkey. Exp Brain Res 118:293-297.

Rand MK, Hikosaka O, Miyachi S, Lu X, Nakamura K, Kitaguchi K, Shimo Y (2000) Characteristics of sequential movements during early learning period in monkeys. Exp Brain Res 131:293-304.

Reynolds C, Ashby P (1999) Inhibition in the human motor cortex is reduced just before a voluntary contraction. Neurology 53:730-735.
Ridding MC, Brouwer B, Nordstrom MA (2000) Reduced interhemispheric inhibition in musicians. Exp Brain Res 133:249-253.

Rossini PM, Marciani MG, Caramia M, Roma V, Zarola F (1985) Nervous propagation along "central" motor pathways in intact man: characteristics of motor responses to "bifocal" and "unifocal" spine and scalp noninvasive stimulation. Electroencephalogr Clin Neurophysiol 61:272-286.

Rossini PM, Caramia MD, Zarola F (1987) Mechanisms of nervous propagation along central motor pathways: noninvasive evaluation in healthy subjects and in patients with neurological disease. Neurosurgery 20:183-191.

Rothwell JC (1997) Techniques and mechanisms of action of transcranial stimulation of the human motor cortex. J Neurosci Methods 74:113-122.

Rouiller EM, Babalian A, Kazennikov O, Moret V, Yu XH, Wiesendanger M (1994) Transcallosal connections of the distal forelimb representations of the primary and supplementary motor cortical areas in macaque monkeys. Exp Brain Res 102:227-243.

Siebner HR, Rothwell J (2003) Transcranial magnetic stimulation: new insights into representational cortical plasticity. Exp Brain Res 148:1-16.

Shim JK, Kim SW, Oh SJ, Kang N, Zatsiorsky VM, Latash ML (2005) Plastic changes in interhemispheric inhibition with practice of a two-hand force production task: a transcranial magnetic stimulation study. Neurosci Lett 374:104-108.

Tettamanti M, Paulesu E, Scifo P, Maravita A, Fazio F, Perani D, Marzi CA (2002) Interhemispheric transmission of visuomotor information in humans: fMRI evidence. J Neurophysiol 88:1051-1058.

Thut G, Halsband U, Regard M, Mayer E, Leenders KL, Landis T (1997) What is the role of the corpus callosum in intermanual transfer of motor skills? A study of three cases with callosal pathology. Exp Brain Res 113:365-370.

Tinazzi M, Zanette G (1998) Modulation of ipsilateral motor cortex in man during unimanual finger movements of different complexities. Neurosci Lett 244:121-124.

Verstynen TD, Konkle T, Ivry RB (2006) Two types of TMS-induced movement variability following stimulation of the primary motor cortex. J Neurophysiol 96:1018-1029.

Waldvogel D, van Gelderen P, Muellbacher W, Ziemann U, Immisch I, Hallett M (2000) The relative metabolic demand of inhibition and excitation. Nature 406:995-998.

Willingham DB (1999) Implicit motor sequence learning is not purely perceptual. Mem Cognit 27:561-572.

Willingham DB, Wells LA, Farrell JM, Stemwedel ME (2000) Implicit motor sequence learning is represented in response locations. Mem Cognit 28:366-375.

Willingham DB, Salidis J, Gabrieli JD (2002) Direct comparison of neural systems mediating conscious and unconscious skill learning. J Neurophysiol 88:1451-1460.

Zhuang P, Dang N, Waziri A, Gerloff C, Cohen LG, Hallett M (1998) Implicit and explicit learning in an auditory serial reaction time task. Acta Neurol Scand 97:131-137.

Ziemann U, Bruns D, Paulus W (1996) Enhancement of human motor cortex inhibition by the dopamine receptor agonist pergolide: evidence from transcranial magnetic stimulation. Neurosci Lett 208:187-190. 